\title{
Baseline characteristics and comparability of older multimorbid patients with polypharmacy and general practitioners participating in a randomized controlled primary care trial
}

Katharina Tabea Jungo ${ }^{1,2}$, Rahel Meier ${ }^{3}$, Fabio Valeri ${ }^{3}$, Nathalie Schwab ${ }^{1,4}$, Claudio Schneider ${ }^{4}$, Emily Reeve ${ }^{5,6}$, Marco Spruit ${ }^{7,8}$, Matthias Schwenkglenks ${ }^{9,10}$, Nicolas Rodondi ${ }^{1,4}$ and Sven Streit ${ }^{1 *}$

\begin{abstract}
Objectives: Recruiting general practitioners (GPS) and their multimorbid older patients for trials is challenging for multiple reasons (e.g., high workload, limited mobility). The comparability of study participants is important for interpreting study findings. This manuscript describes the baseline characteristics of GPs and patients participating in the 'Optimizing PharmacoTherapy in older multimorbid adults In primary CAre' (OPTICA) trial, a study of optimization of pharmacotherapy for multimorbid older adults. The overall aim of this study was to determine if the GPs and patients participating in the OPTICA trial are comparable to the real-world population in Swiss primary care.
\end{abstract}

Design: Analysis of baseline data from GPs and patients in the OPTICA trial and a reference cohort from the FIRE ('Family medicine ICPC Research using Electronic medical records') project.

Setting: Primary care, Switzerland.

Participants: Three hundred twenty-three multimorbid ( $\geq 3$ chronic conditions) patients with polypharmacy $(\geq 5$ regular medications) aged $\geq 65$ years and 43 GPs recruited for the OPTICA trial were compared to 22,907 older multimorbid patients with polypharmacy and 227 GPs from the FIRE database.

Methods: We compared the characteristics of GPs and patients participating in the OPTICA trial with other GPs and other older multimorbid adults with polypharmacy in the FIRE database. We described the baseline willingness to have medications deprescribed of the patients participating in the OPTICA trial using the revised Patients' Attitudes Towards Deprescribing (rPATD) questionnaire.

Results: The GPS in the FIRE project and OPTICA were similar in terms of sociodemographic characteristics and their work as a GP (e.g. aged in their fifties, $\geq 10$ years of experience, $\geq 60 \%$ are self-employed, $\geq 80 \%$ work in a group practice). The median age of patients in the OPTICA trial was 77 years and $45 \%$ of trial participants were women. Patients participating in the OPTICA trial and patients in the FIRE database were comparable in terms of age, certain clinical

\footnotetext{
*Correspondence: sven.streit@biham.unibe.ch

${ }^{1}$ Institute of Primary Health Care (BIHAM), University of Bern, Mittelstrasse

43, 3012 Bern, Switzerland

Full list of author information is available at the end of the article
}

(C) The Author(s) 2021. Open Access This article is licensed under a Creative Commons Attribution 4.0 International License, which permits use, sharing, adaptation, distribution and reproduction in any medium or format, as long as you give appropriate credit to the original author(s) and the source, provide a link to the Creative Commons licence, and indicate if changes were made. The images or other third party material in this article are included in the article's Creative Commons licence, unless indicated otherwise in a credit line to the material. If material is not included in the article's Creative Commons licence and your intended use is not permitted by statutory regulation or exceeds the permitted use, you will need to obtain permission directly from the copyright holder. To view a copy of this licence, visit http://creativecommons.org/licenses/by/4.0/. The Creative Commons Public Domain Dedication waiver (http://creativeco mmons.org/publicdomain/zero/1.0/) applies to the data made available in this article, unless otherwise stated in a credit line to the data. 
characteristics (e.g. systolic blood pressure, body mass index) and health services use (e.g. selected lab and vital data measurements). More than $80 \%$ of older multimorbid patients reported to be willing to stop $\geq 1$ of their medications if their doctor said that this would be possible.

Conclusion: The characteristics of patients and GPs recruited into the OPTICA trial are relatively comparable to characteristics of a real-world Swiss population, which indicates that recruiting a generalizable patient sample is possible in the primary care setting. Multimorbid patients in the OPTICA trial reported a high willingness to have medications deprescribed.

Trial registration: Clinicaltrials.gov (NCT03724539), KOFAM (Swiss national portal) (SNCTP000003060), Universal Trial Number (U1111-1226-8013)

Keywords: Multimorbidity, Polypharmacy, Older adults, General practitioners, Clinical trial, External validity, Baseline characteristics

\section{Introduction}

Globally, the population group of adults aged $\geq 65$ years is growing faster than all other age groups combined. In 2019 one in every 11 persons was 65 years and over, this has been predicted to increase to one in six persons by the year 2050 [1]. With ageing societies, also come growing numbers of older adults with multiple chronic conditions. Multimorbid patients often use multiple medications and with polypharmacy comes a higher risk of using potentially inappropriate medications (PIMs). PIMs are medications for which the risk of potential adverse events outweighs the clinical benefits, such as when there are more effective and safer alternatives available for use in older adults [2]. The use of PIMs is associated with increased risk of adverse drug events, falls and cognitive impairment [3-6]. Patients with multimorbidity and polypharmacy often have complex healthcare needs, which in turn lead to substantial health services use and associated costs [7]. The use of potentially inappropriate medications is high in this patient group [8]. In this context, the 'Optimizing PharmacoTherapy in older multimorbid adults In primary CAre' (OPTICA) trial was launched with the aim of investigating whether an electronic clinical decision support tool can help GPs to optimise medication use of older multimorbid patients with polypharmacy.

Lack of external validity of clinical trials, the extent to which results can be generalised to the wider population, has been cited as a reason that interventions do not get adopted after publication of the study. One factor that can influence external validity is the characteristics of the participants recruited into the trial; that is, whether they are comparable (have similar characteristics) to those found in the real-world population [9].

Despite societal ageing and widespread multimorbidity, patients with chronic conditions and older adults in general are often underrepresented in clinical research $[10,11]$. Evidence from studies of younger and healthier participants may not be generalizable to the broader older multimorbid population [12]. The reasons for the exclusion and general underrepresentation of complex older adults in research are multifaceted. On the one hand, studies often have inclusion and exclusion criteria to maximise participant retention and minimise variability among participants [13-15]. On the other hand, even if older multimorbid adults are not explicitly excluded, major barriers to recruiting this type of study participants include limited mobility (e.g. not being able to attend multiple appointments or complete certain tests), and in the case of cognitive impairment, inability to provide informed consent $[14,16,17]$. Additionally, the person identifying and selecting patients for recruitment (e.g. member of the research team or through healthcare professionals with established relationships) can impact the external validity of participants [18]. Use of routinely collected patient information to identify participants for clinical trials is a promising method to reduce the labour of recruitment. However, concerns exist about the error rate of using electronic medical records for this [19].

Not only can the recruitment of older multimorbid patients be challenging, so can the recruitment of GPs [20]. Previous studies found that time constraints, lack of training, fear of loss of professional autonomy as well as lack of rewards and recognition are barriers to research participation for physicians in general [21]. Conducting clinical research in the primary care setting comes with additional challenges. For instance, a lack of infrastructure, lack of financial remuneration of practice staff involvement, misunderstandings on how daily clinical work in general practice could accommodate the clinical research, and seasonal changes in workload [22, 23]. There is the concern that GPs with specific characteristics or attitudes can be motivated more easily to participate in clinical research. If true, it would mean that the results of an interventional study (such as our OPTICA trial) would not be generalizable to even the local context outside of those who participated in the trial. Overall, little is known about whether it is possible to recruit 
an externally comparable sample of older multimorbid patients and GPs for research in primary care.

Further, past medication optimization interventions in patients with polypharmacy have shown limited effect in changing medication use $[24,25]$ and/or clinical outcomes (e.g. mortality, cognitive decline) [26]. This may be due to patient resistance to medication changes and their unawareness of potentially inappropriate medication use [27]. It is therefore important to consider not only the characteristics of participants, but their attitudes as well.

The 'Family medicine ICPC Research using Electronic medical records' (FIRE) database is the largest Swiss electronic database containing anonymized routine patient data from the electronic medical records in $>10 \%$ of Swiss primary care practices. It also contains information about the GPs who regularly export data from their electronic medical records. The FIRE database therefore provides a unique opportunity to examine the likely external validity of the OPTICA study results to the wider Swiss general population in primary care.

The overall aim of this study was to determine if the GPs and patients participating in the OPTICA trial are comparable to the real-world population in Swiss primary care. We hypothesised that our broad inclusion criteria and support provided to participating GPs would result in recruitment of comparable participants. This information is not only important for interpreting the forthcoming results of the OPTICA trial (i.e. the likely external validity of the study findings), but can also inform the ability to recruit complex older adults for clinical trials in primary care.

Specifically, the aims of this manuscript were to:

1. Describe the baseline characteristics of participants (GPs and older patients with multimorbidity and polypharmacy) recruited to the OPTICA trial.

2. Compare the characteristics of GPs and patients participating in the OPTICA trial with those in the FIRE database.

3. Compare the characteristics of the patients recruited for OPTICA from random screening lists generated from electronic medical records with patients recruited through GP identification of eligible patients.

4. Describe the patients' willingness to have medications deprescribed.

\section{Methods}

\section{Study design and setting}

For this analysis we used baseline data from the ongoing cluster-randomized controlled trial (cRCT) 'Optimizing PharmacoTherapy in older multimorbid patients In primary CAre' (OPTICA). We were able to compate the OPTICA study participants to reference cohorts from the 'Family medicine ICPC Research using Electronic medical records' (FIRE) project database, as all GPs who participated in the OPTICA trial regularly export data to the FIRE project. Details about these two research projects have been reported elsewhere [28, 29].

The FIRE project is the largest Swiss database collecting anonymized routine patient data from the electronic medical records in primary care practices since 2009 [28]. The following information is available in the FIRE database: administrative information (patient, age, and sex), diagnosis codes, laboratory and vital signs measurements, and prescribing information. As of October 2020, the database of the FIRE project contains data from the electronic medical records of more than 680 GPs (about 11\% of all GPs in Switzerland [30]) and more than 830,000 patients (about 10\% of the Swiss population) [31]. All GPs in Switzerland are invited to join the FIRE project if they use an electronic health record (EHR) program that is compatible with exporting anonymized data to the FIRE project.

The OPTICA trial is a cluster-randomized controlled trial, being conducted in primary care in the German speaking part of Switzerland. The aim of the OPTICA trial is to investigate whether the use of an electronic clinical decision support system, namely the 'Systematic Tool to Reduce Inappropriate Prescribing' (STRIP) Assistant [32], improves medication appropriateness compared to a standard care sham intervention in older multimorbid patients with polypharmacy. The STRIP Assistant (STRIPA) is based on the algorithms of the 'Screening Tool to Alert doctors to Right Treatment' (START) and 'Screening Tool of Older Person's Prescriptions' (STOPP) version 2 [33], which are lists of medications generally considered to be inappropriate and appropriate in older adults, respectively [34]. The standard care sham intervention in the control group consists of a medication discussion between GPs and patients in accordance with usual care. The co-primary outcomes of the OPTICA trial are the 'Medication Appropriateness Index' (MAI) and the 'Assessment of underutilization' (AOU) [35-37]. More detailed background information about the OPTICA trial, the study intervention, and the FIRE project is reported in eAppendix 1 in the supplement.

\section{Participants \\ OPTICA trial}

We present the inclusion and exclusion criteria for GPs and patients in the OPTICA trial in Table 1. To maximise the generalizability of the study population, we kept the exclusion criteria to a minimum. Patients were recruited through their GPs. GPs were instructed to use a random screening list generated from the data they exported to 


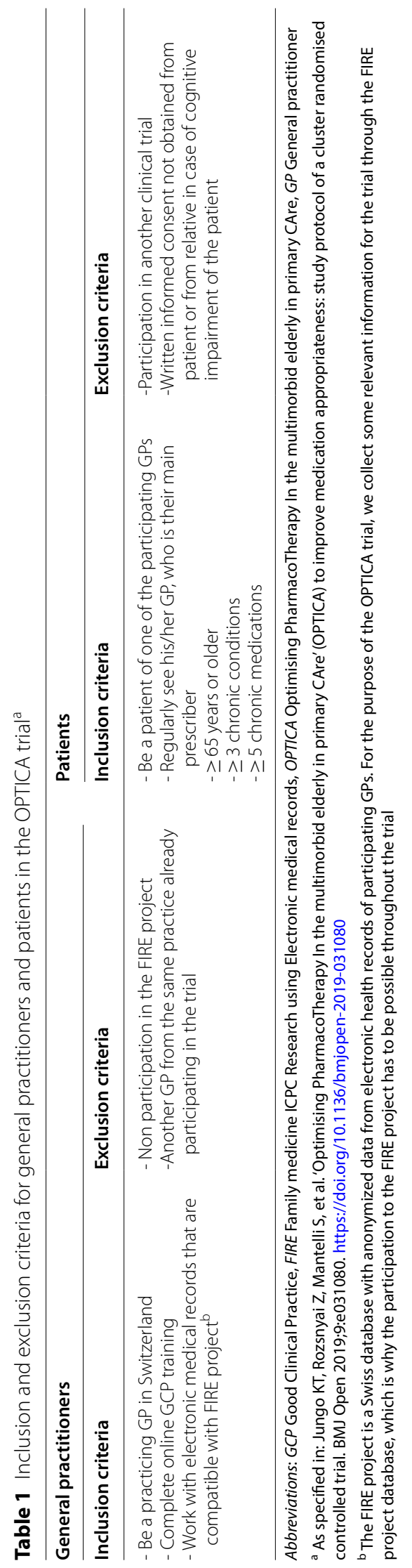


the FIRE project, but also had the flexibility to recruit other eligible patients after exhausting the screening lists. The calculated sample size of the OPTICA trial was 320 patients (details reported in the OPTICA protocol paper [29]).

\section{FIRE project reference cohort}

As of May 2019, around 520 GPs participated in the FIRE project. To define the target population of patients, we identified patients in the FIRE database who were at least 65 years and were prescribed at least 5 different medications at the time point of May $1^{\text {st }}, 2019$. The selection of reference GPs for the analyses took place as follows: GPs participating in the FIRE project, who were the GP of one of the patients included in the patient reference population (as described above) were included in the GP reference cohort $(n=227)$. GPs who participated in FIRE, but did not have any older multimorbid patients with polypharmacy (e.g. because they had only recently joined the project and did not yet export data) and those who took place in the OPTICA trial $(n=43)$ were excluded from the GP reference cohort. eFigure 1 in the supplement visualizes the creation of the reference cohorts.

\section{Data query and variables}

From the FIRE database we extracted patients and GP characteristics. For GPs we extracted sociodemographic information and variables describing their work as GP (as shown in Table 2). For patients we extracted sociodemographic information, clinical parameters and variables describing their health services use (Table 3 ). All variables measuring health services use or reporting vital data and lab values were reported for the period of the last 12 months before May 2019.

The information on patients' willingness to have medications deprescribed was collected in the baseline phone call conducted with participants in the OPTICA trial using the German translation of the revised Patients' Attitudes Towards Deprescribing (rPATD) questionnaire. The original questionnaire was developed by Reeve et al. [38, 40]. The German translation was validated and used in a Swiss study on patients' attitudes towards having medication deprescribed [39]. The rPATD questionnaire for both caregivers and patients contains two global questions as well as questions grouped into four factors: medication burden, mediation appropriateness, concerns about stopping, and involvement. There are four to five questions per factor, which can be used to calculate a factor score. Each factor score ranges from 1 to 5 [40].

\section{Statistical analysis}

First, we compared the characteristics of GPs participating in the OPTICA trial with those of the reference GPs in the FIRE database. Second, we compared the characteristics of the OPTICA study participants with those of other older, multimorbid patients in the FIRE database. Third, we compared the characteristics of the patients recruited for OPTICA from the random screening lists with the OPTICA patients recruited directly by GP identification of eligible participants (i.e. not from the screening lists). Finally, we described patients' willingness to have medications deprescribed. We also performed a sensitivity analysis, by comparing the characteristics of the OPTICA study participants with all other older patients of the same GP only.

Categorical data are presented as frequencies and percentages, and continuous variables as median and interquartile range (IQR), as the variables were nonnormally distributed. For categorical variables we performed a Fisher's exact text and for continuous variables a Kruskal-Wallis test was performed, as defined in the $\mathrm{R}$ package "tableone" [41]. For this study, if the $p$-value was $<0.05$ we concluded that there was sufficient evidence to say that the groups were statistically different. We also calculated standardized differences, which can be used to compare balances in measured variables [42]. While $p$-values were used for the statistical hypothesis testing, absolute standardized difference (ASD) values helped quantify the differences between groups. An ASD value $>0.2$ has previously been defined as representing an imbalance between two groups [43]. Hence for the purpose of this study we considered a maximum threshold of 0.2 for ASD value as being acceptable in terms of comparability of the two groups. The group comparisons were performed using the statistical software package R (Version 3.6.3) [44].

The analyses on patients' willingness to deprescribe were performed using the statistical software Stata 15.1 (StataCorp, College Station, TX, USA). We calculated the four factor scores (involvement, burden, appropriateness, and concerns about stopping) as described previously [40]. Each score is calculated based on responses to the 5 items within each factor of the rPATD questionnaire and ranges from 1-5. In addition, we present the responses to the two stand-alone statements from the rPATD ("Overall, I am satisfied with my current medicines" and "If my doctor said it was possible I would be willing to stop one or more of my regular medicines").

\section{Patient and public involvement}

As described in the OPTICA protocol paper [29], GPs and older patients with multimorbidity and polypharmacy are represented in the independent Safety and Data Monitoring Board of the OPTICA trial. GPs participating in the OPTICA trial receive regular newsletters. 
Table 2 Baseline characteristics of general practitioners in the OPTICA trial compared to the general practitioners in the FIRE database

\begin{tabular}{|c|c|c|c|c|}
\hline Characteristics & OPTICA GPs $(N=43)$ & FIRE GPs $(N=227)^{1}$ & $P$-value ${ }^{5}$ & $\begin{array}{l}\text { Absolute } \\
\text { standardized } \\
\text { difference }^{6}\end{array}$ \\
\hline Median age (IQR) & $54(45-60)$ & $51(44-58)$ & 0.572 & 0.073 \\
\hline Median years since starting to work as GP (IQR) & $15(6-23)$ & $10(5-21)$ & 0.302 & 0.159 \\
\hline \multicolumn{5}{|l|}{ Sex } \\
\hline Women (\%) & $8(19)$ & $80(35)$ & 0.034 & 0.385 \\
\hline Men (\%) & $35(81)$ & $146(65)$ & & \\
\hline \multicolumn{5}{|l|}{ Employment status } \\
\hline Self-employed (\%) & $28(70)$ & $131(63)$ & 0.474 & 0.143 \\
\hline Employed (\%) & $12(30)$ & $76(37)$ & & \\
\hline \multicolumn{5}{|l|}{ GP practice type } \\
\hline Group practice (\%) & $36(84)$ & $200(88)$ & 0.452 & 0.126 \\
\hline Single practice (\%) & $7(16)$ & $27(12)$ & & \\
\hline \multicolumn{5}{|l|}{ Location } \\
\hline Non-urban (\%) & $17(40)$ & $51(23)$ & 0.022 & 0.375 \\
\hline Urban (\%) & $26(60)$ & $176(78)$ & & \\
\hline \multicolumn{5}{|l|}{ Self-dispensation of medications in GP office ${ }^{2}$} \\
\hline Yes (\%) & $25(60)$ & $175(77)$ & 0.046 & 0.386 \\
\hline No (\%) & $13(31)$ & $41(18)$ & & \\
\hline Limited $^{3}(\%)$ & $4(10)$ & $11(5)$ & & \\
\hline Median work percentage (IQR) & $80(80-100)$ & $80(60-100)$ & 0.020 & 0.401 \\
\hline \multicolumn{5}{|l|}{ Participation in integrated care model } \\
\hline Yes & $39(93)$ & $202(95)$ & 0.456 & 0.103 \\
\hline No & $3(7)$ & $10(5)$ & & \\
\hline $\begin{array}{l}\text { Median percentage of eligible patients based on } \\
\text { OPTICA inclusion criteria (IQR) }\end{array}$ & $6(3-14)$ & $7(4-11)$ & 0.614 & 0.287 \\
\hline
\end{tabular}

Abbreviations: GP General practitioner, IQR Interquartile range, OPTICA Optimizing PharmacoTherapy in older multimorbid adults In primary CAre, FIRE Family medicine ICPC Research using Electronic medical records

${ }^{1}$ As of spring May 2019, excludes GPs who were part of the OPTICA trial and who did not have any eligible patients for the OPTICA trial

${ }^{2}$ Depending on the area/region they work in, GPs in Switzerland may be able to sell and dispense medications to their patients

${ }^{3}$ Only for selected medications

${ }^{4} \geq 5$ medications from different ATC groups and age $\geq 65$ years. The other inclusion and exclusion criteria were not implemented, as they had to be double checked by the GPs

${ }^{5}$ For categorical variables we performed a Fisher's exact text and for continuous variables a Kruskal-Wallis test was performed; $P$-values of $<0.05$ represent that there is evidence for a statistically significant difference between the two groups

${ }^{6} \mathrm{An}$ imbalance between the two groups was previously defined as an absolute standardize difference value $>0.2$

At the end of the study, study participants are informed about their study allocation and the results of the study.

\section{Results}

The process of the recruitment of GPs and patients in the OPTICA trial is shown in the trial flow chart (Fig. 1). Out of 121 GPs showing interest in the OPTICA trial, 94 were contacted for a recruitment visit in their GP office (explanation of study design, tasks for participating GPs, and if needed, installation of FIRE data export tools), and 43 were recruited. Out of 934 patients on the screening lists, 224 were recruited. Additionally, 99 patients (30.6\% of the total patients recruited) were recruited through GP identification of eligible patients (outside of the screening list).

\section{What types of GPs participated in the OPTICA trial, and how did they compare to the non-participating GPs from FIRE?}

As shown in Table 2, GPs who participated in OPTICA and those from the FIRE reference cohort were in their fifties on average $($ OPTICA median $=54$, FIRE median $=51)$, had several years of experience working as a GPs (OPTICA median $=15$, FIRE median $=10)$, and the majority were self-employed (OPTICA self-employed $=70 \%$, FIRE selfemployed $=63 \%$ ). The GPs in the FIRE reference cohort and OPTICA were similar in terms of age, median years since starting to work as a GP, employment status, GP practice type, and participation in integrated care models ( $p$-values $>0.05$ and absolute standardized differences $(\mathrm{ASD})<0.2)$. We found differences between OPTICA and 
Table 3 Baseline characteristics of patients in the OPTICA trial compared to other multimorbid patients with polypharmacy in the FIRE database

\begin{tabular}{|c|c|c|c|c|}
\hline Characteristics & $\begin{array}{l}\text { OPTICA study } \\
\text { participants }{ }^{1} \\
(N=323)\end{array}$ & $\begin{array}{l}\text { Patients in the FIRE } \\
\text { database }^{2}\left(N=22^{\prime} 907\right)\end{array}$ & $P$-value ${ }^{3}$ & $\begin{array}{l}\text { Absolute } \\
\text { standardized } \\
\text { difference }^{4}\end{array}$ \\
\hline Median age (IQR) & $77(73-83)$ & $78(72-84)$ & 0.630 & 0.053 \\
\hline \multicolumn{5}{|l|}{ Sex } \\
\hline Women (\%) & $146(45)$ & $12^{\prime} 699(55)$ & 0.001 & 0.206 \\
\hline Men (\%) & $177(55)$ & $10^{\prime} 207(45)$ & & \\
\hline Median number of chronic conditions (IQR) ${ }^{5}$ & $4(3-6)$ & $3(3-5)$ & $<0.001$ & 0.422 \\
\hline Median number of medications in the last 12 months $(\mathrm{IQR})^{6}$ & $6(5-9)$ & $7(5-8)$ & $<0.001$ & 0.23 \\
\hline \multicolumn{5}{|l|}{ Health services use (in the last 12 months) } \\
\hline Median number of consultations (IQR) & $16(10-25)$ & $13(7-22)$ & $<0.001$ & 0.216 \\
\hline Median number of blood pressure measurements (IQR) & $3(2-5)$ & $2(1-4)$ & $<0.001$ & 0.276 \\
\hline Median number of Body Mass Index measurements (IQR) & $2(1-3)$ & $1(1-3)$ & 0.501 & 0.03 \\
\hline Median number of $\mathrm{HbA} 1 \mathrm{c}$ measurements (IQR) & $2(1-4)$ & $2(1-3)$ & 0.001 & 0.24 \\
\hline $\begin{array}{l}\text { Median number of glomerular filtration rate (GFR) measure- } \\
\text { ments (IQR) }\end{array}$ & $2(1-3)$ & $1(1-3)$ & $<0.001$ & 0.208 \\
\hline Median number of lipid profile measurements (IQR) & $1(1-2)$ & $1(1-1)$ & 0.166 & 0.093 \\
\hline \multicolumn{5}{|l|}{ Lab values \& vital signs (in the last 12 months) } \\
\hline Median systolic blood pressure (IQR) & $138(126-148)$ & $138(127-149)$ & 0.541 & 0.023 \\
\hline Median diastolic blood pressure (IQR) & $76(70-83)$ & $79(72-85)$ & 0.005 & 0.154 \\
\hline Median Body Mass Index (IQR) & $29(25-32)$ & $28(24-31)$ & 0.235 & 0.101 \\
\hline Median HbA1c (IQR) & $6.3(5.7-7)$ & $6.1(5.6-6.9)$ & 0.023 & 0.1 \\
\hline Median GFR (IQR) & $66.2(51.4-79.7)$ & $68.3(52.3-82.5)$ & 0.314 & 0.041 \\
\hline
\end{tabular}

Abbreviations: BMI Body Mass Index, IQR Interquartile range, GFR Glomerular filtration rate, HbA1c Hemoglobin A1C, OPTICA Optimizing PharmacoTherapy in older multimorbid adults In primary CAre, FIRE Family medicine ICPC Research using Electronic medical records

${ }^{1}$ Patients who participated in the OPTICA trial

${ }^{2}$ Patients eligible to participate in the OPTICA trial based on the inclusion and exclusion criteria, excludes patients who participated in the OPTICA trial

${ }^{3}$ For categorical variables we performed a Fisher's exact text and for continuous variables a Kruskal-Wallis test was performed. $P$-values of $<0.05$ represent that there is evidence for a statistically significant difference between the two groups

${ }^{4}$ An imbalance between the two groups was previously defined as an absolute standardize difference value $>0.2$

${ }^{5}$ Chronic conditions were defined according to Lamers et al. and O'Halloran et al. [38, 39]

${ }^{6}$ Number of medications belonging to different groups defined by the Anatomical Therapeutic Chemical (ATC) classification system

GPs from the FIRE reference cohort with regards to sex (lower proportion of female GPs in the OPTICA trial), location (greater proportion of OPTICA GPs in nonurban areas), and self-dispensing of medications in GP office (lower proportion of OPTICA GPs than FIRE GPs). The median work percentage was $80 \%$ in both groups (4-day week), but $p$-value and ASD showed that the distribution of the work percentages was different between groups.

\section{What types of patients consented to participate in the OPTICA trial, and how did they compare to non-participants?}

As shown in Table 3, patients participating in the OPTICA trial were relatively comparable to other older patients with multimorbidity in the FIRE reference cohort with regards to their clinical characteristics and health services use. On average, patients were in their late seventies (OPTICA median $=77$, FIRE median $=78$ ), and regularly saw their GP (OPTICA median consultation counts in the last 6 months $=16$, FIRE median $=13$ ). We did not find evidence for a difference between the groups with regards to age, the median number of Body Mass Index (BMI) measurements, the median number of lipid profile measurements, median systolic blood pressure, median BMI and median number of glomerular filtration rate (GFR) measurements ( $p$-values $>0.05$ and ASD $<0.1$ for all these variables). Median diastolic blood pressure and median $\mathrm{HbA} 1 \mathrm{c}$ values were found to be statistically significant between groups, but the ASD was close or equal to 0.1 . For most of the remaining variables, we found statistically significant differences and standardized differences of around 20\% (e.g. sex, median number of consultations, median number of medications, etc.). On average, 
General practitioners (GPs) showing interest in participating in the OPTICA trial $(n=121)$

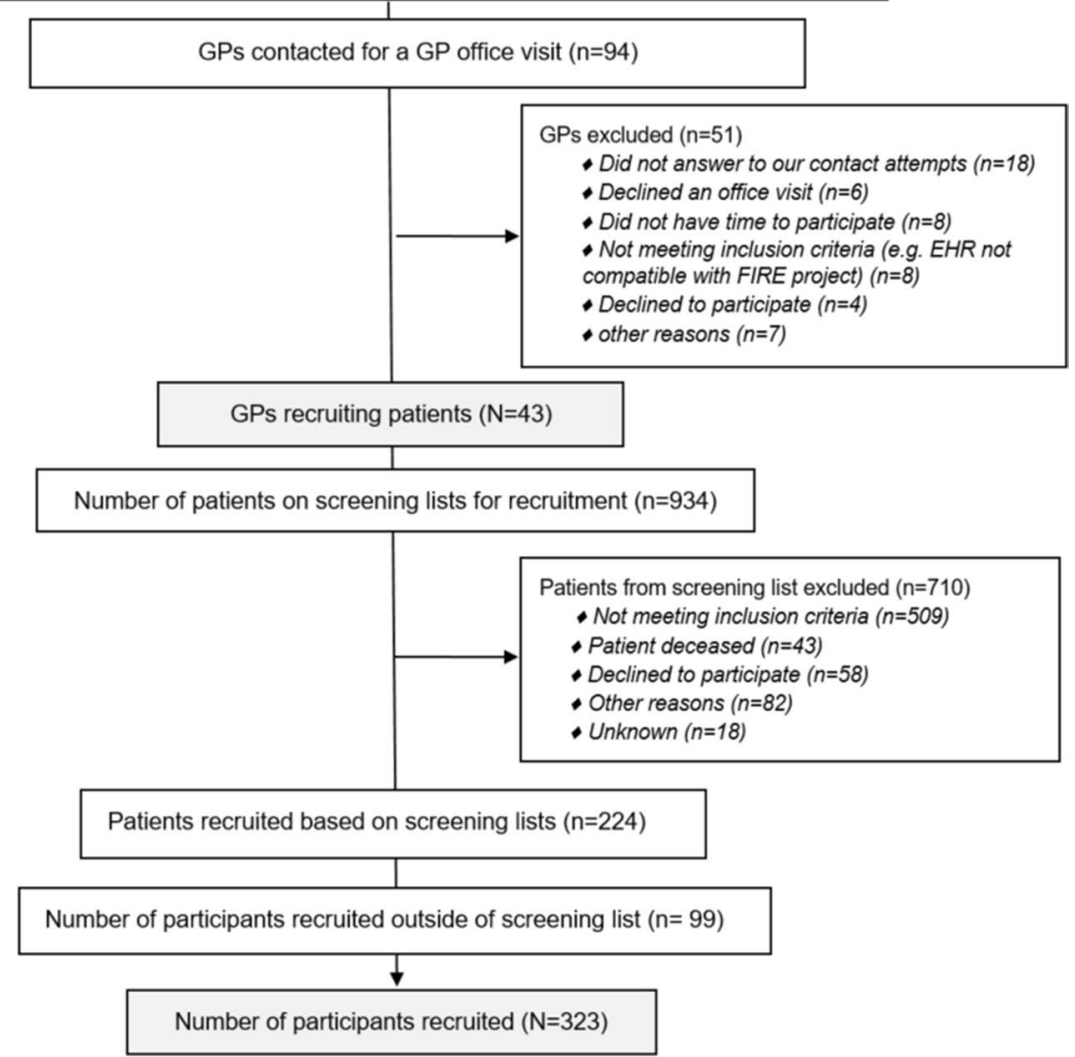

Fig. 1 Flow chart of recruitment of general practitioners and patients in the OPTICA trial'. 'cluster-randomized controlled trial in Swiss primary care. Abbreviations: OPTICA = Optimizing PharmacoTherapy in older multimorbid adults In primary CAre

patients in the OPTICA trial had more chronic conditions (OPTICA median $=4$, FIRE median $=3, \mathrm{ASD}=0.422$ ), but less medications (OPTICA median $=6$, FIRE median $=7$, $\mathrm{ASD}=0.23$ ). Within patients of the same GP, patients participating in OPTICA were comparable to patients not participating in OPTICA (eTable 1 in the supplement).

\section{How did patients recruited from random screening lists and other patients compare?}

Two hundred and twenty-four patients were recruited from the random screening lists and 99 patients were recruited outside of these lists. The comparison of these two group (Table 4) found that they were comparable. We only found a statistically significant difference concerning median number of consultations $(p=0.031)$ and number of BMI measurements $(p=0.022)$.

\section{What was study participants' willingness to have medications deprescribed?}

As shown in Table 5, at baseline of the OPTICA trial, the majority of patients in the OPTICA trial (>90\%) reported to be satisfied with their current medications. Furthermore, most of the study participants (>80\%) reported to be willing to stop one or more of their medications if their doctor said that it was possible. The OPTICA study participants reported to be involved in their medication use (median involvement score $=4.8 \quad(\mathrm{IQR}=4.2-5.0)$; score can range from 1 to 5 , with 5 representing a high reported involvement). The median medication burden score was $2.2(\mathrm{IQR}=1.6-2.8)$ and the concerns about stopping score was $1.6(\mathrm{IQR}=1.0-2.4)$. Results of caregivers who completed the caregiver rPATD (where the patient was unable to complete the questionnaire due to cognitive impairment, $n=16$ ) are shown in Table 5 . 
Table 4 Baseline characteristics of patients in the OPTICA trial who were recruited from the screening list and those who were recruited outside of the screening list

\begin{tabular}{|c|c|c|c|c|}
\hline Characteristics & $\begin{array}{l}\text { OPTICA study participants } \\
\text { from screening list }(N=224)\end{array}$ & $\begin{array}{l}\text { OPTICA study participants } \\
\text { not from screening list } \\
(N=99)\end{array}$ & $P$-value ${ }^{1}$ & $\begin{array}{l}\text { Absolute } \\
\text { standardized } \\
\text { difference }^{2}\end{array}$ \\
\hline Median age (IQR) & $77(72-82)$ & $79(74-84)$ & 0.088 & 0.183 \\
\hline \multicolumn{5}{|l|}{ Sex } \\
\hline Women (\%) & $106(47)$ & $40(40)$ & 0.276 & 0.14 \\
\hline Men (\%) & $118(53)$ & $59(60)$ & & \\
\hline Median number of chronic conditions (IQR) & $4(3-6)$ & $4(3-6)$ & 0.774 & 0.086 \\
\hline $\begin{array}{l}\text { Median number of medications in the last } 12 \text { months } \\
\text { (IQR) }\end{array}$ & $6(5-9)$ & $7(3-9)$ & 0.464 & 0.16 \\
\hline \multicolumn{5}{|l|}{ Health services use (in the last 12 months) } \\
\hline Median number of consultations (IQR) & $17(10-26)$ & $14(9-21)$ & 0.031 & 0.303 \\
\hline $\begin{array}{l}\text { Median number of blood pressure measurements } \\
\text { (IQR) }\end{array}$ & $3(2-6)$ & $3(1-5)$ & 0.197 & 0.034 \\
\hline $\begin{array}{l}\text { Median number of Body Mass Index measurements } \\
(\mathrm{IQR})\end{array}$ & $1(1-2)$ & $2(1-3)$ & 0.255 & 0.329 \\
\hline Median number of $\mathrm{HbA} 1 \mathrm{c}$ measurements (IQR) & $2(1-3)$ & $2(1-4)$ & 0.332 & 0.147 \\
\hline $\begin{array}{l}\text { Median number of glomerular filtration rate (GFR) } \\
\text { measurements (IQR) }\end{array}$ & $2(1-3)$ & $2(1-3)$ & 0.901 & 0.045 \\
\hline Median number of lipid profile measurements (IQR) & $1(1-2)$ & $1(1-2)$ & 0.667 & 0.101 \\
\hline \multicolumn{5}{|l|}{ Lab values \& vital signs (in the last 12 months) } \\
\hline Median systolic blood pressure (IQR) & $137(125-147)$ & $139(130-150)$ & 0.397 & 0.102 \\
\hline Median diastolic blood pressure (IQR) & $76(70-83)$ & $76(71-83)$ & 0.801 & 0.078 \\
\hline Median Body Mass Index (IQR) & $29(25-32)$ & $29(25-33)$ & 0.902 & 0.015 \\
\hline Median HbA1c (IQR) & $6.3(5.8-7.0)$ & $6.4(5.6-7.0)$ & 0.991 & 0.02 \\
\hline Median GFR (IQR) & $66.5(53.4-80.1)$ & $62.7(48-6-78.9)$ & 0.264 & 0.167 \\
\hline
\end{tabular}

Abbreviations: BMI Body Mass Index, IQR Interquartile range, GFR Glomerular filtration rate, HbA1C Hemoglobin A1C, OPTICA Optimizing PharmacoTherapy in older multimorbid adults In primary CAre

${ }^{1}$ For categorical variables we performed a Fisher's exact text and for continuous variables a Kruskal-Wallis test was performed. $P$-values of $<0.05$ represent that there is evidence for a statistically significant difference between the two groups

${ }^{2}$ An imbalance between the two groups was previously defined as an absolute standardize difference value $>0.2$

\section{Discussion}

To inform the likely external validity of the results of the OPTICA trial, we compared the characteristics of our participating GPs and patients to a Swiss real-world reference cohort. We also examined the characteristics of patients recruited based on random screening lists (created from electronic medical records) and those recruited outside of these lists by their GP to see whether a bias in the selection may exist. Finally we explored the willingness of patients in OPTICA to have medications deprescribed which allows us to reflect on the possible impact that this may have on the outcomes of the trial and compare them to previously studied populations. From our analyses we have some confidence that the findings of the OPTICA study will be generalizable to the broad Swiss population of GPs and patients. We found that the GPs in the FIRE project and OPTICA were similar in terms of sociodemographic characteristics and their work as a GP (e.g. age, experience as GP, employment status, and GP practice type). We also found that patients participating in the OPTICA trial and patients in the FIRE database were comparable in terms of age, median number of certain lab and vital data measurements (e.g. BMI, lipid profile, GFR measurements) and certain clinical characteristics (e.g. systolic blood pressure, BMI). For the variables that differed between the two groups according to the statistical tests, the absolute standardized differences were generally around 0.2 (or 20\%), with an imbalance of the two groups having previously been defined as $>0.2$. Patients who participated in the OPTICA trial reported a high level of willingness to stop one or more of their medications.

Overall, our study results showed that GPs who participated in the OPTICA trial and those who participated in the FIRE project were comparable in most of the variables examined. Previous research showed that high performing physicians are more likely to participate in research [45]. When looking at the patient data, we observed that OPTICA patients had more chronic conditions, but less medications. The absolute standardized differences indicate some imbalances between the groups on these variables. 
Table 5 Patients' and caregivers' willingness to have medications deprescribed assessed with 'revised Patients' Attitudes Towards Deprescribing' (rPATD) questionnaire

\begin{tabular}{|c|c|c|}
\hline & $\begin{array}{l}\text { OPTICA } \\
\text { patients } \\
(n=298)\end{array}$ & $\begin{array}{l}\text { Caregivers of OPTICA participants } \\
\text { with cognitive impairment }(n=16)\end{array}$ \\
\hline \multicolumn{3}{|c|}{ "Overall, I am satisfied with my current medicines" (\%) and respectively "Overall, I am satisfied with my care recipient's current medicines" } \\
\hline Strongly agree & $215(72.2)$ & $11(68.7)$ \\
\hline Agree & $64(21.5)$ & $5(31.3)$ \\
\hline Unsure & $4(1.3)$ & - \\
\hline Disagree & $11(3.7)$ & - \\
\hline Strongly disagree & $4(1.3)$ & - \\
\hline \multicolumn{3}{|c|}{$\begin{array}{l}\text { "If my doctor said it was possible I would be willing to stop one or more of my regular medicines" (\%) and respectively "If their doctor said it was pos- } \\
\text { sible I would be willing to stop one or more of my care recipient's medicines" }\end{array}$} \\
\hline Strongly agree & $224(75.2)$ & $10(62.5)$ \\
\hline Agree & $38(12.8)$ & $3(18.8)$ \\
\hline Unsure & $9(3.0)$ & $1(6.3)$ \\
\hline Disagree & $14(4.7)$ & $1(6.3)$ \\
\hline Strongly disagree & $13(4.4)$ & $1(6.3)$ \\
\hline \multicolumn{3}{|l|}{ Factor scores } \\
\hline Involvement: Median involvement in medication management score (IQR) & $4.8(4.2-5.0)$ & $4(3.4-5.0)$ \\
\hline \multicolumn{3}{|c|}{$\begin{array}{l}\text { [range: } 1-5 \text {, the higher the score the more 'involved' patients are with their medications and caregivers with the medications of the person they care } \\
\text { for] }\end{array}$} \\
\hline Burden: Median perceived burden of medications score (IQR) & $2.2(1.6-2.8)$ & $2.3(1.3-3.8)$ \\
\hline \multicolumn{3}{|c|}{ [range: $1-5$, the higher the score the more burdensome patients and caregivers perceive/view/experience the medications to be] } \\
\hline Appropriateness: Median belief in appropriateness of medications score (IQR) & $3.8(3.4-4.2)$ & $3.8(3.4-4.2)$ \\
\hline \multicolumn{3}{|c|}{ [range: $1-5$, the higher the score the more appropriate patients respectively caregivers perceive/view/experience the medications] } \\
\hline Concerns about stopping: Median concerns about stopping medications score (IQR) & $1.6(1.0-2.4)$ & $1.2(0.8-1.6)$ \\
\hline
\end{tabular}

Abbreviations: OPTICA Optimizing PharmacoTherapy in older multimorbid adults In primary CAre, rPATD Revised Patients' Attitudes Towards Deprescribing

${ }^{a}$ Reeve, E., Low, L. F., Shakib, S., \& Hilmer, S. N. (2016). Development and Validation of the Revised Patients' Attitudes Towards Deprescribing (rPATD) Questionnaire: Versions for Older Adults and Caregivers. Drugs \& Aging, 33(12), 913-928. Since the scores were not normally distributed we decided to present the medians

While one can argue about whether the differences are clinically relevant, this observation could indicate that GPs in the OPTICA trial may have been more proactive in reviewing the medications of their patients than other GPs. If the latter was the case, this would mean that the intervention of the OPTICA trial may be limited in its effect (i.e. if the patients had little room for further optimisation of their medications). We also found differences in sex, location and self-dispensing between GPs in both groups. These differences may have stemmed from the recruitment strategy used in the OPTICA trial, which in the context of difficulties of recruiting GPs for clinical research focused (and therefore needing to optimise GP recruitment) did not specifically recruit based on their baseline characteristics. The sex composition of the OPTICA GPs could affect the final results, since female physicians have been found to be less likely to make deprescribing decisions [46].

We found that the multimorbid older patients who participated in the OPTICA trial were comparable to those in the FIRE database in terms of sociodemographic variables, health services use and clinical characteristics. For the variables were there was a statistically significant difference between the groups, most had standardized differences close but not passing the ASD threshold of 0.2 for meaningful differences between the groups (e.g. number of medications: OPTICA median $=6$, FIRE median $=7, \mathrm{ASD}=0.23$, number of consultations OPTICA median $=16$, FIRE median $=13, \mathrm{ASD}=0.216$ ). There is a lower proportion of female participants in the OPTICA trial than in the reference FIRE cohort. However, since no difference in willingness to deprescribe according to sex has been identified $[39,47]$, we do not anticipate that this sex imbalance will affect the results of the OPTICA trial.

We found that the trial participants recruited from the random screening lists (around two thirds of patients) and those who were recruited outside of these lists (around one third of patients) were comparable. While systematic differences in recruitment behaviour (i.e. differential recruitment [48]) has been reported previously in the context of a cluster-randomized controlled trial in primary care (UK BEAM trial) [49], we did not find evidence for a bias in the selection of participants in the OPTICA trial. 
The UK BEAM trial reported, for example, that patients in participating practices were experiencing milder back pain (which the intervention targeted) than those in the control group and thus highlighted the potential for the recruitment process to bias study results [49]. The use of random screening lists helped to standardize patient recruitment but, in light of the imperfect nature of the screening lists, we also allowed GPs to recruit patients who were not on these lists. We assumed that giving participating GPs some flexibility in the recruitment process would allow them to better integrate recruitment into their regular practice and would therefore optimise recruitment.

Concerning patients' willingness to deprescribe, we found that the OPTICA study participants had a high involvement in their medication use and $>80 \%$ were willing to stop one or more of their regular medications if their doctor told them this was possible. These findings are in line with previous research. Another study conducted in Switzerland found that $77 \%$ of older adults would be willing to stop one or more of their medications [39] and similar proportions were found in studies in other countries (88\% in Australia [47], 92\% in the United States [50], 83\% in Singapore [51]). While these numbers have to be interpreted with caution (e.g. social desirability bias, not medication specific, hypothetical nature of the question), it shows that older patients may be open to optimizing their medication use through deprescribing. We also found the factor scores to be comparable to the results from a study in Australian older adults [47]. This information is crucial for implementing medication optimization interventions, and in the context of the OPTICA trial, it shows that patients' attitudes towards deprescribing may not be a barrier to implementation of deprescribing.

While the results presented in this manuscript are primarily Swiss-specific, we can draw a more broadly applicable conclusion; it appears to be possible to recruit a sample of study participants in primary care trials that is comparable to real life cohorts.

\section{Strengths \& limitations}

The OPTICA trial had a low number of exclusion criteria, which facilitated broad recruitment of study participants. However, the analyses in this manuscript have several limitations. First, in Switzerland there are no complete GP or patient registries. The FIRE project maintains the only primary care database in Switzerland of this size, but it does not include all GPs in Switzerland, and in turn, does not include all patients in Switzerland. In Switzerland, not all GPs use electronic health record programs. The use of electronic health records in Switzerland increased from around 40 to $>70 \%$ from 2012 to 2020 [17, 34], but remains lower than in other high-income countries. Furthermore, not all GPs who fulfil the eligibility criteria self-select to participate in the FIRE project. This raises the question of the representativeness of the GPs in the FIRE database. However, two recent assessments of the Swiss GP workforce showed that the GPs in the FIRE project are comparable to the entire GP workforce in terms of age, sex, experience as GP and work percentage (eTable 2 in the supplement). These similarities between OPTICA, FIRE and all GPs in Switzerland signify that the recruitment of an externally comparable sample of GPs is possible in randomized clinical trials in the Swiss primary care setting. This confirms previous evidence from the UK, which showed that achieving good levels of external validity was possible in clinical trials in primary care [52]. However, due to the lack of patient registries, we cannot comment on the comparability of patients in the FIRE project and Swiss patients in general. While the analyses presented in this manuscript do not confirm external validity of the forthcoming OPTICA trial results, they do facilitate future interpretation of our findings.

Next, inherent to routine medical databases, like the FIRE database, is a certain risk of information bias and missing data as information is only collected when it is clinically relevant [53]. Since we used data from before the OPTICA study intervention started, we assume that both our groups would have been affected by the same potential sources of bias. Despite the similarities found between the FIRE and Swiss GP workforce in terms of sociodemographic and work-related characteristics, we were unable to compare other important characteristics between the two groups (e.g. quality of care, relationship and trust between doctor and patient). Our finding that the patients included in the OPTICA trial had less medication but more chronic conditions than the reference cohort could reflect the selection of "good performers" which may bias the findings of the OPTICA trial. Our analysis of patients' willingness to deprescribe was limited to patients in the OPTICA trial and could not be compared directly to the reference cohort and this questionnaire is not used in regular clinical care. Other limitations related to the rPATD are that it asks hypothetical questions, it is not specific to certain medications, and it might be subject to social desirability bias. Furthermore, for the purpose of the OPTICA trial the rPATD was translated from English to German; back-translation and piloting was conducted to increase the validity of the translation, but other measures of validation and reliability of the translation in the local context were not conducted (e.g. test-retest reliability). Finally, due to the uncertainties surrounding the absolute standardized differences, we decided to present both $p$-values and ASD. While there may be debate of the cut off to use for ASD, we used $>0.2$ as this has been recommended by Yang et Dalton [42, 43]. If we considered a smaller threshold, such as $>0.1$, 
it would not have changed our conclusions about the groups being comparable.

\section{Conclusion}

In the OPTICA trial, it was possible to recruit GPs and their older patients with multimorbidity and polypharmacy that are generally comparable to a real-world reference cohort of GPs and older patients with multimorbidity and polypharmacy in Switzerland. The observed similarities between OPTICA, FIRE and all Swiss GPs signify that the recruitment of an externally valid sample of GPs is possible in randomized clinical trials in the Swiss primary care setting. The findings from this manuscript about the baseline characteristics of study participants will be crucial for interpreting the wider applicability of the OPTICA study intervention and its findings. Ensuring that clinical trials recruit comparable populations is crucial for improving the care of older multimorbid patients, which have previously been underrepresented in clinical research.

\begin{abstract}
Abbreviations
ASD: Absolute standardized difference; FIRE: 'Family medicine ICPC Research using Electronic medical records'; GCP: Good Clinical Practice; GP: General practitioner; OPTICA: Optimising PharmacoTherapy In the multimorbid elderly in primary CAre; rPATD: Revised Patients' Attitudes Towards Deprescribing.
\end{abstract}

\section{Supplementary Information}

The online version contains supplementary material available at https://doi. org/10.1186/s12875-021-01488-8.

Additional file 1: eAppendix 1. Information about the OPTICA trial and the FIRE project. eTable 1. Baseline characteristics of patients in the OPTICA trial compared to other multimorbid patients with polypharmacy in the FIRE database who also were patients of the general practitioners participating in the OPTICA trial. eTable 2. Baseline characteristics of Swiss general practitioners who participated in the Workforce-Study. eFigure 1. FIRE database, FIRE reference cohorts and OPTICA trial participants.

\section{Acknowledgements \\ Thanks to Heinz Bhend, Pius Bürki, and Sven Trelle for their support in conducting the OPTICA trial. We would also like to thank all GPs and their patients for participating in the OPTICA trial for all their efforts to help us conduct this trial. And finally, we would like to thank Stéphanie Giezendanner and Andreas Zeller for providing information on the Swiss GP workforce.}

\section{Ethics and dissemination}

The ethics committee of the canton of Bern in Switzerland approved the protocol of the OPTICA trial. The Ethics Committee of the Canton of Zurich approved studies within the FIRE project. OPTICA embraces an open access policy and will vigorously disseminate all resulting data, study results and publications.

\section{Authors' contributions}

Study concept and design: All authors (KTJ, RM, FV, NS, CS, ER, MSp, MSch, NR, SS). Statistical analysis: KTJ, RM. Drafting of the manuscript: KTJ, RM, SS. Critical revision of the manuscript for important intellectual content: all authors (KTJ, RM, FV, NS, CS, ER, MSp, MSch, NR, SS). Obtained funding: NR, SS. Administrative, technical, or material support: NS, SS. Supervision: SS. All authors read and approved the final manuscript.

\section{Funding}

This work is supported by the Swiss National Science Foundation (SNSF), within the framework of the National Research Programme 74 (NRP74) "smarter healthcare" (project number 407440_167465 to SS, NR, and MS). ER is supported by an Australian NHMRC-ARC Dementia Research Development Fellowship (APP1105777).

\section{Availability of data and materials}

The FIRE database can be accessed at any time by the scientific team of the institute. For external requests, access has to be requested from the head of the institute.

\section{Declarations}

Ethics approval and consent to participate

The ethics committee of the canton of Bern (Switzerland) and the Swiss regulatory authority (Swissmedic) approved the study protocol of the OPTCIA trial and other documentation on study conduct (BASEC ID: 2018-00914). The ethics committee and Swissmedic receive annual safety reports and information about study stops/end and protocol amendments, as per local requirements. Written informed consent was obtained from all study participants participating in the OPTICA trial. The OPTICA trial was performed in accordance with relevant guidelines and regulations. The local Ethics Committee of the Canton of Zurich approved studies within the FIRE project database (BASEC-Nr. Req-2017-00797). Due to the anonymized nature of the EHR data in the FIRE database individual patient consent was not required. The analyses presented in this manuscript did not require an additional ethical approval.

\section{Consent for publication}

Not applicable.

\section{Competing interests}

The authors do not have any conflicts of interest to declare.

\section{Author details}

${ }^{1}$ Institute of Primary Health Care (BIHAM), University of Bern, Mittelstrasse 43, 3012 Bern, Switzerland. ${ }^{2}$ Graduate School for Health Sciences, University of Bern, Bern, Switzerland. ${ }^{3}$ Institute of Primary Care, University and University Hospital of Zurich, Zurich, Switzerland. ${ }^{4}$ Department of General Internal Medicine, Inselspital, Bern University Hospital, University of Bern, Bern, Switzerland. ${ }^{5}$ Quality Use of Medicines and Pharmacy Research Centre, UniSA: Clinical and Health Sciences, University of South Australia, Adelaide, South Australia, Australia. ${ }^{6}$ Geriatric Medicine Research, Faculty of Medicine and College of Pharmacy, Dalhousie University and Nova Scotia Health Authority, Halifax, NS, Canada. ${ }^{7}$ Department of Information and Computing Sciences, Utrecht University, Utrecht, The Netherlands. ${ }^{8}$ Public Health \& Primary Care, Leiden University Medical Centre, Leiden University, Leiden, The Netherlands. ${ }^{9}$ Institute of Pharmaceutical Medicine (ECPM), University of Basel, Basel, Switzerland. ${ }^{10}$ Epidemiology, Biostatistics and Prevention Institute, University of Zurich, Zurich, Switzerland.

Received: 12 January 2021 Accepted: 9 June 2021

Published online: 22 June 2021

\section{References}

1. United Nations. Department of Economics and Social Affairs. Population dynamics. 2019 revision of world population prospects 2019. Available from: https://population.un.org/wpp/. Accessed 28 May 2021.

2. Fu AZ, Liu GG, Christensen DB. Inappropriate medication use and health outcomes in the elderly. J Am Geriatr Soc. 2004;52(11):1934-9. 
3. Xing $X X$, Zhu C, Liang HY, Wang K, Chu YQ, Zhao LB, et al. Associations between potentially inappropriate medications and adverse health outcomes in the elderly: a systematic review and meta-analysis. Ann Pharmacother. 2019;53(10):1005-19.

4. Masumoto S, Sato M, Maeno T, Ichinohe Y, Maeno T. Potentially inappropriate medications with polypharmacy increase the risk of falls in older Japanese patients: 1-year prospective cohort study. Geriatr Gerontol Int 2018;18(7):1064-70.

5. Koyama A, Steinman M, Ensrud K, Hillier TA, Yaffe K. Long-term cognitive and functional effects of potentially inappropriate medications in older women. J Gerontol A Biol Sci Med Sci. 2014;69(4):423-9.

6. Liew TM, Lee CS, Goh Shawn KL, Chang ZY. Potentially inappropriate prescribing among older persons: a meta-analysis of observational studies. Ann Fam Med. 2019;17(3):257-66.

7. Bähler C, Huber CA, Brüngger B, Reich O. Multimorbidity, health care utilization and costs in an elderly community-dwelling population: a claims data based observational study. BMC Health Serv Res. 2015;15(1):23.

8. Jungo KT, Streit S, Lauffenburger JC. Utilization and spending on potentially inappropriate medications by US older adults with multiple chronic conditions using multiple medications. Arch Gerontol Geriatr. 2021;93:104326

9. Rothwell PM. External validity of randomised controlled trials: "to whom do the results of this trial apply?" Lancet. 2005;365(9453):82-93.

10. Jadad AR, To MJ, Emara M, Jones J. Consideration of multiple chronic diseases in randomized controlled trials. JAMA. 2011;306(24):2670-2.

11. Shenoy $P$, Harugeri A. Elderly patients' participation in clinical trials. Perspect Clin Res. 2015;6(4):184-9.

12. Golomb BA, Chan VT, Evans MA, Koperski S, White HL, Criqui MH. The older the better: are elderly study participants more non-representative? A cross-sectional analysis of clinical trial and observational study samples. BMJ Open. 2012;2(6):e000833.

13. Buffel du Vaure C, Dechartres A, Battin C, Ravaud P, Boutron I. Exclusion of patients with concomitant chronic conditions in ongoing randomised controlled trials targeting 10 common chronic conditions and registered at ClinicalTrials.gov: a systematic review of registration details. BMJ Open. 2016;6(9):e012265.

14. McMurdo ME, Witham MD, Gillespie ND. Including older people in clinical research. BMJ. 2005;331(7524):1036-7.

15. Reeve E, Trenaman SC, Rockwood K, Hilmer SN. Pharmacokinetic and pharmacodynamic alterations in older people with dementia. Expert Opin Drug Metab Toxicol. 2017;13(6):651-68.

16. Forsat ND, Palmowski A, Palmowski Y, Boers M, Buttgereit F. Recruitment and retention of older people in clinical research: a systematic literature review. J Am Geriatr Soc. 2020;68(12):2955-63.

17. Mody L, Miller DK, McGloin JM, Freeman M, Marcantonio ER, Magaziner $\mathrm{J}$, et al. Recruitment and retention of older adults in aging research. J Am Geriatr Soc. 2008:56(12):2340-8.

18. Mannel RS, Walker JL, Gould N, Scribner DR Jr, Kamelle S, Tillmanns T, et al. Impact of individual physicians on enrollment of patients into clinical trials. Am J Clin Oncol. 2003;26(2):171-3.

19. Köpcke F, Prokosch HU. Employing computers for the recruitment into clinical trials: a comprehensive systematic review. J Med Internet Res. 2014;16(7):e161.

20. Tognoni G, Alli C, Avanzini F, Bettelli G, Colombo F, Corso R, et al. Randomised clinical trials in general practice: lessons from a failure. BMJ. 1991;303(6808):969-71.

21. Ross S, Grant A, Counsell C, Gillespie W, Russell I, Prescott R. Barriers to participation in randomised controlled trials: a systematic review. J Clin Epidemiol. 1999;52(12):1143-56.

22. Ward $\mathrm{E}$, King M, Lloyd M, Bower P, Friedli K. Conducting randomized trials in general practice: methodological and practical issues. Br J Gen Pract. 1999:49(448):919-22.

23. Leathem CS, Cupples ME, Byrne MC, O'Malley M, Houlihan A, Murphy AW, et al. Identifying strategies to maximise recruitment and retention of practices and patients in a multicentre randomised controlled trial of an intervention to optimise secondary prevention for coronary heart disease in primary care. BMC Med Res Methodol. 2009;9:40.

24. Kouladjian O'Donnell L, Gnjidic D, Sawan M, Reeve E, Kelly PJ, Chen $T F$, et al. Impact of the goal-directed medication review electronic decision support system on drug burden index: a cluster-randomised clinical trial in primary care. Br J Clin Pharmacol. 2020;87(3):1499-511.

25. O'Mahony D, Gudmundsson A, Soiza RL, Petrovic M, Jose CruzJentoft $A$, Cherubini $A$, et al. Prevention of adverse drug reactions in hospitalized older patients with multi-morbidity and polypharmacy: the SENATOR* randomized controlled clinical trial. Age Ageing. 2020;49(4):605-14

26. Huiskes VJB, Burger DM, van den Ende CHM, van den Bemt BJF. Effectiveness of medication review: a systematic review and meta-analysis of randomized controlled trials. BMC Fam Pract. 2017;18(1):5.

27. Achterhof AB, Rozsnyai Z, Reeve E, Jungo KT, Floriani C, Poortvliet RKE, et al. Potentially inappropriate medication and attitudes of older adults towards deprescribing. PLoS One. 2020;15(10):e0240463.

28. Chmiel C, Bhend H, Senn O, Zoller M, Rosemann T. The FIRE project: a milestone for research in primary care in Switzerland. Swiss Med Wkly. 2011;140:w13142.

29. Jungo KT, Rozsnyai Z, Mantelli S, Floriani C, Löwe AL, Lindemann F, et al. 'Optimising PharmacoTherapy In the multimorbid elderly in primary CAre' (OPTICA) to improve medication appropriateness: study protocol of a cluster randomised controlled trial. BMJ Open. 2019:9(9):e031080.

30. mfe Haus- und Kinderärzte Schweiz. Hausärztemangel - aber mit Licht am Horizont: Workforce-Studie 2020 - das Wichtigste in Kürze 2020. Available from: https://www.mfe-standpunkte.ch/de/ausgabe/ausga be-22020--43/artikel/hausaerztemangel-aber-mit-licht-am-horizont76. Accessed 28 May 2021

31. Institut für Hausarztmedizin IHAMZ. FIRE-Daten 2019. Available from: https://www.hausarztmedizin.uzh.ch/de/fire2/welchedatenbeinhalte tdasprojekt.html. Accessed 28 May 2021.

32. Shen Z, Meulendijk M, Spruit M. A federated information architecture for multinational clinical trials: STRIPA revisited. Istanbul: 24th European Conference on Information Systems (ECIS 2016); 2016.

33. Huibers CJA, Sallevelt BTGM, de Groot DA, Boer MJ, van Campen JPCM, Davids CJ, et al. Conversion of STOPP/START version 2 into coded algorithms for software implementation: a multidisciplinary consensus procedure. Int J Med Informatics. 2019:125:110-7.

34. O'Mahony D, O'Sullivan D, Byrne S, O'Connor MN, Ryan C, Gallagher P. STOPP/START criteria for potentially inappropriate prescribing in older people: version 2. Age Ageing. 2015;44(2):213-8.

35. Hanlon JT, Schmader KE, Ruby CM, Weinberger M. Suboptimal prescribing in older inpatients and outpatients. J Am Geriatr Soc. 2001:49(2):200-9.

36. Hanlon JT, Schmader KE, Samsa GP, Weinberger M, Uttech KM, Lewis IK, et al. A method for assessing drug therapy appropriateness. J Clin Epidemiol. 1992;45(10):1045-51.

37. Samsa GP, Hanlon JT, Schmader KE, Weinberger M, Clipp EC, Uttech $\mathrm{KM}$, et al. A summated score for the medication appropriateness index: development and assessment of clinimetric properties including content validity. J Clin Epidemiol. 1994;47(8):891-6.

38. Reeve E, Anthony AC, Kouladjian O'Donnell L, Low LF, Ogle SJ, Glendenning JE, et al. Development and pilot testing of the revised Patients' Attitudes Towards Deprescribing questionnaire for people with cognitive impairment. Australas J Ageing. 2018;37(4):E150-4.

39. Rozsnyai Z, Jungo KT, Reeve E, Poortvliet RKE, Rodondi N, Gussekloo J, et al. What do older adults with multimorbidity and polypharmacy think about deprescribing? The LESS study - a primary care-based survey. BMC Geriatr. 2020;20(1):435.

40. Reeve E, Low LF, Shakib S, Hilmer SN. Development and validation of the Revised Patients' Attitudes Towards Deprescribing (rPATD) questionnaire: versions for older adults and caregivers. Drugs Aging. 2016;33(12):913-28.

41. Kazuki Y. Introduction to tableone 2020. Available from: https://cran.rproject.org/web/packages/tableone/vignettes/introduction.html. Accessed 28 May 2021.

42. Austin PC. Balance diagnostics for comparing the distribution of baseline covariates between treatment groups in propensity-score matched samples. Stat Med. 2009;28(25):3083-107.

43. Yang D, Dalton J, editors. A unified approach to measuring the effect size between two groups using SAS. 2012.

44. R Core Team. R: a language and environment for statistical computing. Vienna: R Foundation for Statistical Computing; 2019. 
45. Dahrouge S, Armstrong CD, Hogg W, Singh J, Liddy C. High-performing physicians are more likely to participate in a research study: findings from a quality improvement study. BMC Med Res Methodol. 2019;19(1):171.

46. Jungo KT, Mantelli S, Rozsnyai Z, ..., Streit S. General practitioners' deprescribing decisions in older adults with polypharmacy: a case vignette study in 31 countries. BMC Geriatr. 2021;21(19):1-12.

47. Reeve E, Low LF, Hilmer SN. Attitudes of older adults and caregivers in Australia toward deprescribing. J Am Geriatr Soc. 2019;67(6):1204-10.

48. Shi Y, Cameron CJ, Heckathorn DD. Model-based and design-based inference: reducing bias due to differential recruitment in respondent-driven sampling. Sociol Methods Res. 2019;48(1):3-33.

49. Farrin A, Russell I, Torgerson D, Underwood M. Differential recruitment in a cluster randomized trial in primary care: the experience of the UK back pain, exercise, active management and manipulation (UK BEAM) feasibility study. Clin Trials (London, England). 2005;2(2):119-24.

50. Reeve E, Wolff JL, Skehan M, Bayliss EA, Hilmer SN, Boyd CM. Assessment of attitudes toward deprescribing in older medicare beneficiaries in the United States. JAMA Intern Med. 2018;178(12):1673-80.

51. Kua CH, Reeve E, Ratnasingam V, Mak VSL, Lee SWH. Patients' and caregivers' attitudes towards deprescribing in Singapore. J Gerontol A Biol Sci Med Sci. 2020;76(6):1053-60.

52. Chaplin K, Bower P, Man M-S, Brookes ST, Gaunt D, Guthrie B, et al. Understanding usual care for patients with multimorbidity: baseline data from a cluster-randomised trial of the 3D intervention in primary care. BMJ Open. 2018;8(8):e019845.

53. Rassen JA, Bartels DB, Schneeweiss S, Patrick AR, Murk W. Measuring prevalence and incidence of chronic conditions in claims and electronic health record databases. Clin Epidemiol. 2019;11:1-15.

\section{Publisher's Note}

Springer Nature remains neutral with regard to jurisdictional claims in published maps and institutional affiliations.

Ready to submit your research? Choose BMC and benefit from:

- fast, convenient online submission

- thorough peer review by experienced researchers in your field

- rapid publication on acceptance

- support for research data, including large and complex data types

- gold Open Access which fosters wider collaboration and increased citations

- maximum visibility for your research: over $100 \mathrm{M}$ website views per year

At BMC, research is always in progress.

Learn more biomedcentral.com/submissions 